\title{
Introduction: Classical and quantum plasmas-matter under extreme conditions
}

\author{
Vincenzo Aquilanti ${ }^{1}$. Mario Capitelli ${ }^{2}$. Francesco Pegoraro ${ }^{3,4}$
}

Published online: 14 February 2019

๑) Accademia Nazionale dei Lincei 2019

The international conference "Classical and quantum plasmas: matter under extreme conditions" was held on 5-6 April, 2018 at the Accademia Nazionale dei Lincei in Rome organized with the contribution of Fondazione Donegani. This interdisciplinary conference addressed the physics and the chemistry of plasmas in different regimes of particle density, of electron energy and of magnetic field. Specific themes of investigation were selected, covering the statistical mechanics foundations of plasmas as many-body classical and quantum systems with long range interactions and the chemistry of collisional plasmas dominated by the Boltzmann equation. Open problems that are common to the dynamics of plasmas from chemical and physical perspectives, such as the roles of collective behaviour and of energy transfer processes, were discussed. It was stressed that this latter aspect is essential for plasma chemistry, being strongly linked to the dynamics of elementary physicochemical processes. The opportunity was taken in some of the talks to evoke the six decades long list of contributions initiated by

This editorial is an introduction to a collection of peer reviewed papers presented at the Conference "Classical and quantum plasmas: matter under extreme conditions" held at Accademia Nazionale dei Lincei in Rome on April 5-6, 2018.

Vincenzo Aquilanti

vincenzoaquilanti@yahoo.it

Mario Capitelli

mario.capitelli@ nanotec.cnr.it

Francesco Pegoraro

pegoraro@df.unipi.it

1 Dipartimento di Chimica, Università di Perugia, Via Elce di Sotto 8, 06123 Perugia, Italy

2 Nanotec CNR, Via Amendola 122/D, 70126 Bari, Italy

3 Physics Department, Pisa University, largo Pontecorvo 3, 56127 Pisa, Italy

4 National Research Council, National Institute of Optics, via G. Moruzzi 1, Pisa, Italy the schools of Ettore Molinari (Plasma Chemistry) and of Gian Gualberto Volpi (Molecular Dynamics) (Fig. 1).

"The unusual properties of plasmas": this is the title of two seminars that the Italian physicist E. Persico ${ }^{1}$ gave in Palermo and then in Catania in April 1959. The notes he prepared for those seminars can be accessed ${ }^{2}$ and contain statements that maintain their full validity after almost sixty years. One such sentence (in translation as for the title of the seminars) reads: "This branch of physics is characterized by the fact that, although the laws of the microscopic elementary phenomena are known (Electromagnetism and Mechanics), these phenomena are so intertwined that the prediction and the interpretation of the macroscopic phenomena are often extremely difficult".

Although presented as a difficulty, this statement identifies one of the main interesting features of plasmas i.e. that a reductionist approach that tries to describe the phenomenology of a system simply as the sum of elementary processes does not fit well the complexity of the dynamics of plasmas and does not account for its variety in the different regimes that are encountered in the Universe and in the laboratory. In fact, quoting now from the document "Plasma at the frontier of scientific discovery" by the US Department of Energy, ${ }^{3}$ "Plasma phenomena occur throughout the Universe, in laboratory and natural settings, covering an enormous range of scales and parameters. For example, spatial scales extend from nanoscale radiation sources to galactic scale magnetic fields, while temporal scales range from attosecond X-ray laser plasma interactions to dynamo magnetic fields varying over centuries. Parameters such as temperature and density

\footnotetext{
${ }^{1}$ Enrico Persico (August 9, 1900-June 17, 1969). Together with E. Amaldi, Persico understood the importance of the research into controlled thermonuclear fusion and helped its birth in Italy at the end of the 50 s.

2 Università degli Studi di Roma, "La Sapienza" Dipartimento di Fisica, Fondo: Enrico Persico, Serie 3: attività scientifica, Sottoserie 5: Lezioni e conferenze 73.

${ }^{3}$ Office of Science, US Department of Energy (2015) https://scien ce.energy.gov/ /media/fes/pdf/program-news/Frontiers_of_Plasma_ Science_Final_Report.pdf.
} 
Fig. 1 Left: Electron phase space at the break of a one dimensinal Langmuir wave in a relativistic plasma (Simulation performed with a Vlasov code developed by A. Grassi at Pisa University). Right: Vlasov simulation of two dimensional magnetized plasma turbulence, performed at CINECA on Marconi super-computer (Courtesy of F. Califano and the PRACE 2017174107 group)

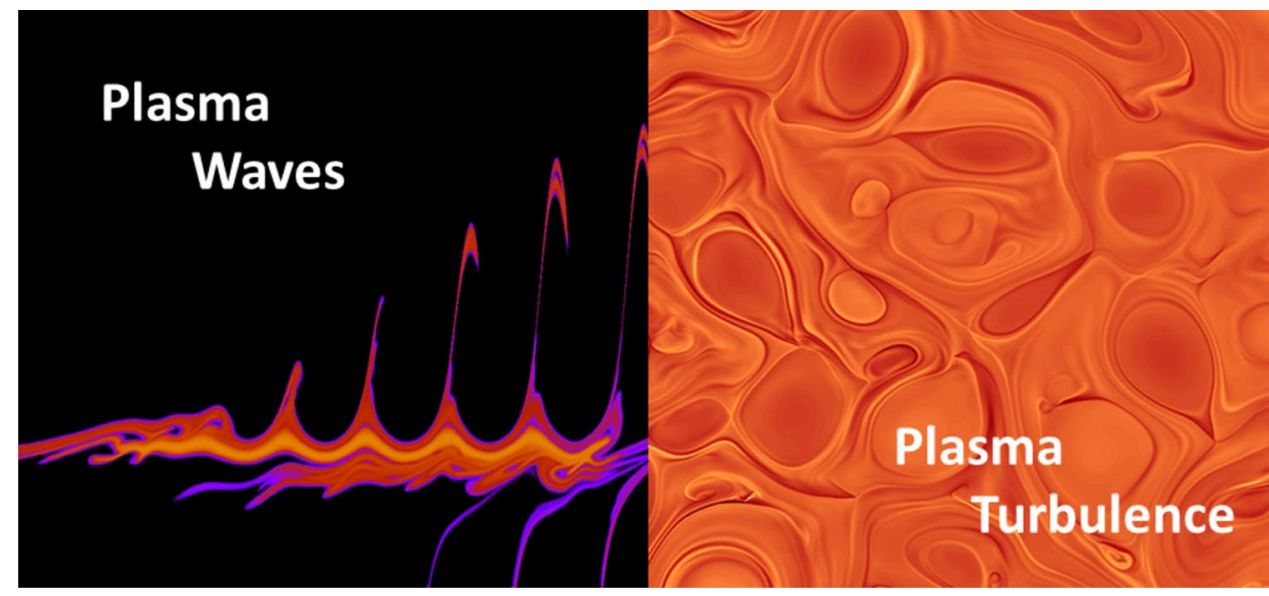

vary over more than 10 and 25 orders of magnitude, respectively. Nevertheless, plasmas exhibit common physical phenomena over these enormous ranges."

The considerations reported above refer to high energy plasmas even though to a certain extent they could be applied to the so called "cold" plasmas i.e. to plasmas in which the electron temperature is much higher than the translational temperature of the heavy components (neutrals and ions). A characteristic feature of the latter case concerns the occurrence of non Maxwellian electron energy distribution functions as well as non equilibrium internal (vibrational and electronic) distribution functions. This does preclude the very definition of the concept of temperature which, in some cases, loses any meaning. Under these conditions extensive use is made of the Boltzmann equation as well as of a PICMCC (Particle in Cell with Montecarlo collisions) methodology to characterize the different situations that may occur in several applied fields. $\dot{A}$ characteristic of these plasmas is the essential role played by collisions in shaping the different non equilibrium distributions as well as in promoting chemical reactions that involve heavy-particle-heavy-particle collisions and electron-heavy-particle collisions.

The conference started by addressing recent developments in the study of high temperature, high energy plasmas in the laboratory and in space, of the interaction of plasmas with coherent electromagnetic fields and of collective phenomena in Bose-Einstein condensates.

F. Pegoraro introduced the subject of relativistic plasmas stressing that novel developments are expected as the investigation of relativistic plasmas in the laboratory approaches the conditions where Quantum Electrodynamic effects start to play an important role. V. Y. Fortov discussed the effects of non-ideality and of degeneracy covering a range of phenomena that spans from inertial fusion plasmas to plasma astrophysics and cosmology, to the interior of large planets. S. V. Bulanov presented an overview of the physical processes that occur in a plasma under the action of a relativistically strong radiation generated by high power lasers, from the nonlinear waves for the creation of compact electron accelerators to the generation of coherent hard electromagnetic radiation can be generated in the form of high harmonics. L. Pitaevski discussed magnetic solitons in a Bose-Einstein condensate and described the new and unusual phenomena that can be observed in the presence of a coherent Rabi coupling between the spin states. A. Di Piazza discussed multiparticle and coherence effects within strong-field Quantum Electrodynamics, indicating that in such regimes there is no easy way to disentangle collective effects from quantum effects. Moving to the astrophysics context, M. Tavani reviewed the main properties of transient plasma phenomena in Galactic sources, active galaxies hosting black holes, and in gamma-ray bursts. Finally, M. Nisoli described recent experimental and theoretical results in the generation of attosecond light pulses. These will offer the opportunity to investigate unexplored features of electron dynamics in complex molecules and solids with unprecedented time resolution.

The "cold" plasma part of the meeting included the discussion of results for elementary processes, non equilibrium internal and thermal distributions and plasma kinetics. Quantum, classical and semiclassical dynamics methods are used to obtain cross sections of elementary processes to be employed to construct predictive models for many plasma chemistry applications.

V. Aquilanti focused on elementary processes at the microscopic scale obtained using mass spectrometry and molecular beam experimental techniques and by computer assisted classical and quantum simulation. Among the many results obtained by the Perugia school he highlighted the phenomenological characterization of intermolecular forces and the acquisition of detailed information regarding various series of elementary reactions relevant to many disciplines, including the new science of astrochemistry.

A. Laganà and F. Esposito investigated cross sections and rate coefficients of heavy particle-heavy particle collisions as a function of the vibrational levels of partners. P. 
Casavecchia discussed molecular crossed beam and laser techniques for determining reaction rates of elementary processes, to be used in particular in combustion and plasma chemistry. The accuracy of cross sections of elementary processes depend on the potential energy surface (PES) of the interacting partners. Very accurate PES can be obtained by quantum chemistry as well as using phenomenological approaches. This latter aspect was discussed by F. Pirani and A. Laricchiuta and applied to the estimation of transport cross sections of atomic and molecular species in high temperature planetary atmospheres.

Non equilibrium internal and thermal distributions are described by statistical mechanics as well as by the Fokker Planck (F-P) equation. E. Son described the requirements for non equilibrium conditions to exist conditions when the tails of the relevant distributions become inverse power laws. S.Willitsch showed that an ion in a radiofrequency ion trap interacting with a buffer gas of ultracold neutral atoms develops a non-thermal distribution with a power law tail. Finally, S. Longo applied a time dependent solution of the F-P equation obtained using the Diffusion Monte Carlo (DMC) method to describe the non equilibrium vibrational distribution of the asymmetric $\mathrm{CO}_{2}$ mode.

Plasma kinetics included selfconsistent models (sm) based on the coupling of electron energy distribution function and state to state kinetics of excited states and global models (gm) which involve important aspects, such as diffusion and plasma-wall reactor interaction. The contributions of M. Capitelli and D. Pietanza for the plasma activation of $\mathrm{CO}_{2}$ and of $\mathrm{G}$. Colonna for plasma assisted combustion are typical examples of (sm) models. Global models were presented by $\mathrm{C}$. Cavallotti to describe the reaction kinetics in non thermal plasmas of $\mathrm{CH}_{4}$ in combustion reactors and by $\mathrm{K}$. Hassouni to discuss dust formation in low power density discharges.

Important experimental contributions were also reported. A. Vanni and F. Taccogna presented the experimental and theoretical development of the ITER Neutral Beam Injector (NBI) for magnetic fusion. Complex network theory and a PIC-MCC methodology were used to allow the identification of the main processes affecting the generation, extraction and acceleration of negative ions and their quantification. G. Isella reported a low energy plasma-enhanced epitaxial growth of germanium micro-crystals on a patterned silicon substrate, while G. Bruno and P. Capezzuto described plasma-chemistry experiments that effectively function as "point-defect healing" processes resulting in high quality layers of graphene.

These results, ${ }^{4}$ as well as those published in this topical issue, show the extraordinary multidisciplinarity of the different subjects. The high energy plasma contributions make wide use of relativistic concepts, non linear plasma dynamics, strong-field quantum electrodynamics, magnetic field reconnection, transient plasma phenomena in Galactic sources and generation of attosecond light pulses. Modern concepts of plasma physics, plasma chemistry and dynamics of elementary processes are used and their dependence on disciplines such as quantum physics, quantum chemistry and statistical mechanics clearly appears.

\footnotetext{
4 The abstracts of all contributions presented during the meeting "Classical and Quantum Plasmas: matter under extreme conditions" are available on the Lincei website http://www.lincei.it/modul es.php name $=$ Convegni\&file $=$ lista $\&$ func $=$ Convegni_edit $\& I d=1492$.
} 